\title{
Effect of stroke thrombolysis predicted by distal vessel occlusion detection
}

Wolfgang G. Kunz, MD, Matthias P. Fabritius, MD, Wieland H. Sommer, MD, MPH, Christopher Höhne, MD, Pierre Scheffler, MD, Lukas T. Rotkopf, Wolfgang P. Fendler, MD, Bastian O. Sabel, MD, Felix G. Meinel, MD, Franziska Dorn, MD, Birgit Ertl-Wagner, MD, MHBA, Maximilian F. Reiser, MD, and

Kolja M. Thierfelder, MD, MSc

Neurology ${ }^{\circledR}$ 2018;90:e1742-e1750. doi:10.1212/WNL.0000000000005519

\section{Abstract \\ Objective}

Among ischemic stroke patients with negative CT angiography (CTA), we aimed to determine the predictive value of enhanced distal vessel occlusion detection using CT perfusion postprocessing (waveletCTA) for the treatment effect of IV thrombolysis (IVT).

\section{Methods}

Patients were selected from 1,851 consecutive patients who had undergone CT perfusion. Inclusion criteria were (1) significant cerebral blood flow (CBF) deficit, (2) no occlusion on CTA, and (3) infarction confirmed on follow-up. Favorable morphologic response was defined as smaller values of final infarction volume divided by initial CBF deficit volume (FIV/CBF). Favorable functional outcome was defined as modified Rankin Scale score of $\leq 2$ after 90 days and decrease in NIH Stroke Scale score of $\geq 3$ from admission to 24 hours ( $\triangle$ NIHSS).

\section{Results}

Among patients with negative CTA $(\mathrm{n}=107)$, $58(54 \%)$ showed a distal occlusion on waveletCTA. There was no difference between patients receiving IVT $(n=57)$ vs supportive care $(\mathrm{n}=50)$ regarding symptom onset, early ischemic changes, perfusion mismatch, or admission NIHSS score (all $p>0.05$ ). In IVT-treated patients, the presence of an occlusion was an independent predictor of a favorable morphologic response (FIV/CBF: $\beta-1.43$; 95\% confidence interval $[\mathrm{CI}]-1.96,-0.83 ; p=0.001$ ) and functional outcome (90-day modified Rankin Scale: odds ratio $7.68 ; 95 \%$ CI $4.33-11.51 ; p=0.039 ; \triangle N I H S S$ : odds ratio $5.76 ; 95 \% \mathrm{CI}$ $3.98-8.27$; $p=0.013$ ), while it did not predict outcome in patients receiving supportive care (all $p>0.05)$.

\section{Conclusion}

In stroke patients with negative CTA, distal vessel occlusions as detected by waveletCTA are an independent predictor of a favorable response to IVT.

\author{
Correspondence \\ Dr. Kunz \\ wolfgang.kunz@med.Imu.de
}




\section{Glossary}

$\mathbf{C B F}=$ cerebral blood flow; $\mathbf{C B V}=$ cerebral blood volume; $\mathbf{C I}=$ confidence interval; $\mathbf{C T A}=\mathrm{CT}$ angiography; $\mathbf{C T P}=\mathrm{CT}$ perfusion; FIV = final infarction volume; IVT = IV thrombolysis; $\mathbf{m R S}=$ modified Rankin Scale; NCCT = noncontrast CT; NIHSS = NIH Stroke Scale; OR = odds ratio; SC = supportive care; waveletCTA = CT perfusion-based wavelet-transformed angiography.

IV thrombolysis (IVT) has been the mainstay of ischemic stroke therapy since $1995 .{ }^{1}$ Its goal is reperfusion of ischemic tissue by targeting occluded arteries, usually detected by $\mathrm{CT}$ angiography (CTA). While some studies showed benefits of IVT in patients without occlusions on CTA, ${ }^{2,3}$ other studies did not. ${ }^{4,5}$ Several recent studies demonstrated larger treatment effects of IVT in patients with vessel occlusions. ${ }^{4-6}$

The function of IVT relates to recanalization. However, recanalization does not always lead to reperfusion as suggested by preclinical ${ }^{7}$ and clinical observations. ${ }^{8-10}$ The no-reflow phenomenon refers to persisting perfusion lesions despite macrovascular recanalization and may be caused by microvascular obstruction. ${ }^{7,11-13}$ As spontaneous recanalization occurs, ${ }^{14,15}$ improved detection of blood clots as IVT targets may be important. Despite growing evidence, occlusions are not considered decision-relevant for IVT by current recommendations. ${ }^{16,17}$

Angiography examinations are routinely performed as singlephase CTA to detect proximal occlusions, which are required for thrombectomy. ${ }^{18}$ However, drawbacks in CTA include low contrast-to-noise ratio, ${ }^{19}$ venous superimposition, ${ }^{20}$ and scan timing imprecisions, ${ }^{21}$ increasing the risk of missing vessel occlusions. Approximately one-third of centers routinely use CT perfusion (CTP) ${ }^{22}$ a dynamic acquisition of the brain. To overcome some CTA limitations, efforts were made to reconstruct angiographic data from CTP. ${ }^{19,23}$ Among these, CTP-based wavelet-transformed angiography (waveletCTA) enables distal occlusion detection in about $50 \%$ of patients without CTA evidence of occlusion but infarction confirmed on follow-up. ${ }^{24}$

Therefore, our aim was to determine the predictive value of distal vessel occlusions detected by waveletCTA in patients with ischemic stroke but negative CTA.

\section{Methods}

\section{Standard protocol approvals, registrations, and patient consents}

The institutional review board of the LMU Munich (Ethikkommission der Medizinischen Fakultät der Ludwig-Maximilians-Universität München) approved this retrospective study, which was conducted according to the Helsinki Declaration of 2013, and waived requirement for informed consent. Based on a prospectively collected stroke registry, our initial cohort consisted of 1,851 consecutive patients who had undergone multiparametric CT including CTP for suspected ischemic stroke between December 2012 and December 2016.

From this cohort, we included all patients with (1) significant cerebral blood flow (CBF) deficits on CTP, (2) missing evidence of vessel occlusion on CTA, and (3) acute ischemic infarction as confirmed by follow-up MRI or CT within 72 hours. We excluded patients with (1) nondiagnostic quality or incomplete raw datasets of CTP or CTA, (2) lacunar etiology of stroke, and (3) hemodynamic infarction pattern.

A significant CBF deficit was defined as the presence of a focal decrease in the color-coded CBF maps compared to the contralateral side on a minimum of 2 adjacent slices. Nineteen CTP examinations had to be excluded based on nondiagnostic CTP quality or incomplete raw CTP datasets. Among 1,832 diagnostic CTP examinations, we excluded 955 patients without CBF deficits, 655 patients with CTAdetected occlusions, 45 patients because of missing follow-up imaging, 29 patients because of missing infarction on followup imaging, 35 patients with confirmed lacunar stroke, and 6 patients with confirmed hemodynamic stroke. The flowchart of patient selection is shown in figure 1. For the functional outcome analysis, patients with prestroke disability as assessed by the premorbid modified Rankin Scale (mRS) with scores $>1$ and patients with missing clinical follow-up were excluded.

\section{CT examination protocol, image postprocessing, and wavelet transform}

All patients underwent standardized CT protocols consisting of noncontrast CT (NCCT), CTA, and CTP (the detailed acquisition protocol has been previously described ${ }^{24}$ ). We used the angiographic reconstruction of waveletCTA from CTP as described in detail and used in previous studies. ${ }^{24-26}$ Briefly, the principle of the algorithm is an analysis of each voxel's time attenuation curve with respect to its resemblance to the shape of a generic contrast bolus time attenuation curve. The wavelet transform then translates this resemblance into a power spectrum, of which the maximal values are interpreted as the angiographic signal intensity. An illustration of the angiographic reconstruction is provided in figure e-1 (links.lww.com/WNL/A439).

\section{Image analysis}

Early ischemic changes on NCCT were assessed using the Alberta Stroke Program Early CT Score ${ }^{27}$ in the case of middle cerebral artery infarction. As there are no established 


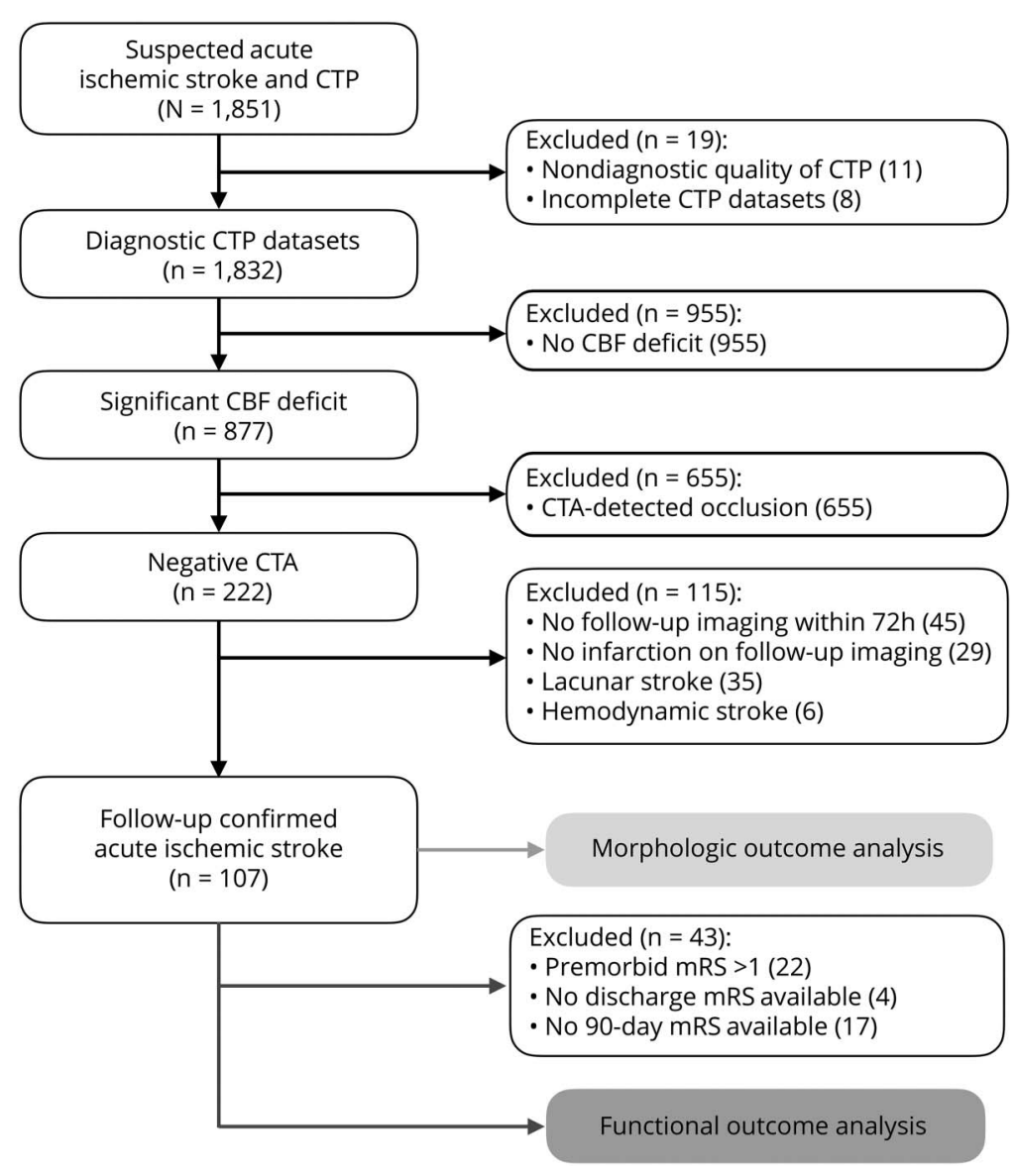

$\mathrm{CBF}=$ cerebral blood flow; $\mathrm{CTA}=\mathrm{CT}$ angiography; $\mathrm{CTP}=\mathrm{CT}$ perfusion; $\mathrm{mRS}=$ modified Rankin Scale. scores for other vascular areas outside the middle cerebral artery territory, we assessed such cases in a binary fashion determining either presence or absence of early ischemic changes. ${ }^{28}$ All CTA examinations of the initial study cohort were reviewed in detail for vessel occlusions using multiplanar reconstructions with the option of maximum intensity projections by 2 experienced radiologists, a boardcertified attending (W.H.S.) with more than 10 years of experience and a board-certified fellow (K.M.T.) with more than 6 years of experience in stroke imaging. CBF deficit, cerebral blood volume (CBV) deficit, and final infarction were volumetrically measured based on expert delineation using OsiriX v.8.0.2 imaging software (Pixmeo, Bernex, Switzerland) as previously described, ${ }^{29}$ an approach that has been shown to be reliable and valid. ${ }^{29-31}$ CBF-CBV mismatch was calculated as absolute volume ( $\mathrm{CBF}$ deficit volume - CBV deficit volume) and as relative mismatch ([CBF deficit volume - $\mathrm{CBV}$ deficit volume $] / \mathrm{CBF}$ deficit volume). Final infarction volume (FIV) was assessed using diffusionweighted MRI $\left(b=1,000 \mathrm{~s} / \mathrm{mm}^{2}\right)$ or NCCT. Two readers independently evaluated the waveletCTA images for vessel occlusions and their location. Readers were blinded to all clinical data and any other imaging studies or reconstructions.

\section{Morphologic and functional outcome analysis}

The primary morphologic outcome parameter was FIV. As secondary morphologic outcome, we determined parameters that represent the morphologic course from ischemia to infarction as applied in previous studies, ${ }^{32-35}$ indicating the morphologic response to treatment. To quantify the change from initial hemodynamic impairment to final infarcted tissue, we used 3 calculated parameters as surrogates: FIV divided by the $\mathrm{CBF}$ deficit volume (FIV/CBF) is a ratio of infarction to the initial ischemia. FIV divided by CBV deficit volume (FIV/ $\mathrm{CBV}$ ) represents a ratio of infarction to initial ischemic core. FIV minus the ischemic core volume divided by the ischemic penumbra volume $([\mathrm{FIV}-\mathrm{CBV}] /[\mathrm{CBF}-\mathrm{CBV}])$ indicates the progression of the ischemic area to the infarcted penumbra. For all 3 parameters alike, smaller values imply more favorable morphologic outcomes.

The functional outcome was assessed using the mRS score upon discharge and after 90 days. In addition, the NIH Stroke Scale (NIHSS) score was assessed on admission and after 24 hours. Ninety-day mRS score was defined as the primary functional outcome parameter. Discharge mRS score and change of the NIHSS score from admission to 24 hours $(\Delta$ NIHSS $\geq 3$ ) as a marker of early neurologic improvement 
were used as secondary functional outcome parameters. Prestroke disability was also evaluated using the mRS. Clinical reasons for withholding IVT treatment are supplied in table e-1 (links.lww.com/WNL/A440).

\section{Statistical analysis}

We performed all statistical analyses using SPSS Statistics 23 (IBM, Armonk, NY). Interreader agreement was assessed using Cohen $\kappa$. The study population was first stratified in 4 subgroups according to treatment status and detection of vessel occlusions. A $\chi^{2}$ test was performed for the group comparison of categorical variables. In case of continuous variables, group comparisons were analyzed by analysis of variance; in case of ordinal variables, a Kruskal-Wallis test was used. Multivariate linear and binary logistic regression analyses were performed to test the association between predictors and the dependent morphologic and functional outcome variables. These analyses were adjusted for age, sex, NIHSS score on admission, early ischemic changes on NCCT, CBF deficit volume, $\mathrm{CBV}$ deficit volume, as well as the relative $\mathrm{CBF}-\mathrm{CBV}$ mismatch. All metric and ordinal variables are presented as median (interquartile range). Normal distribution was assessed using the Kolmogorov-Smirnov test. Categorical variables are presented as frequency and percentage. Two-sided $p$ values $<0.05$ were considered to indicate statistical significance.

\section{Data availability}

Anonymized data will be shared by request from any qualified investigator.

\section{Results}

\section{Patient characteristics}

The study population of 107 patients was analyzed in subgroups according to the treatment status (IVT vs supportive care $[\mathrm{SC}]$ ) and presence of a vessel occlusion as detected by waveletCTA (occlusion vs no occlusion). The detailed patient characteristics are shown in table 1. Overall, in 58 (54\%) of all patients with negative CTA, an occlusion was detected using waveletCTA. The interreader agreement of vessel occlusion detection was very good (Cohen $\kappa=0.864)$.

IVT was administered in 57 patients (53\%); the remaining 50 patients received SC. The detected vessel occlusions showed similar distribution across vascular segments in IVT- and SC-treated patients (all with $p>0.05$ ). Group comparisons showed no differences in age, sex, time from symptom onset, presence of early ischemic changes, Alberta Stroke Program Early CT Score, relative CBF-CBV mismatch, or admission NIHSS score (all with $p>0.05$ ). Occlusions detected by waveletCTA were not associated with the premorbid or admission functional status (all with $p>0.05)$. Differences among the groups were observed for CBF deficit volume, primary morphologic outcome parameters (FIV), as well as primary (90-day mRS score) and secondary functional outcome parameters (discharge mRS score, $\Delta$ NIHSS) (all with $p<0.05$ ). Regression analyses were performed for further statistical analyses regarding independent associations with morphologic and functional outcome parameters.

\section{Predictors of morphologic outcome}

In both treatment groups, no association of waveletCTAdetected occlusions with the primary outcome parameter FIV was present (all with $p>0.05$ ); a moderate association for a favorable outcome was observed within the IVTtreated group $(\beta-7.43 ; 95 \%$ confidence interval $[\mathrm{CI}]$ $-14.86,1.82 ; p=0.107)$. In patients receiving IVT, the presence of waveletCTA-detected occlusions demonstrated an association with smaller values of the morphologic response parameters FIV/CBF $(\beta-1.43$; $95 \%$ CI -1.96 , $-0.83 ; p=0.001)$ and $(\mathrm{FIV}-\mathrm{CBV}) /(\mathrm{CBF}-\mathrm{CBV})(\beta$ -4.54 ; $95 \%$ CI $-7.02,-2.07 ; p=0.001)$, indicating a favorable response, whereas no associations were found for patients who received SC (all with $p>0.05$ ). The results of the regression analysis are presented in table 2 . Representative patient examples for the IVT treatment response are shown in figure 2. Graphic representations of the morphologic outcome parameters are provided in figures e- 2 to e-4 (links.lww.com/WNL/A439).

\section{Predictors of functional outcome}

In patients receiving IVT, associations for the presence of a waveletCTA-detected occlusion with favorable primary (90-day $\mathrm{mRS}$ score $\leq 2$ : odds ratio [OR] 7.68; 95\% CI $4.33-11.51 ; p=0.039)$ and secondary functional outcome parameters (discharge mRS score $\leq 2$ : OR 8.33 ; $95 \% \mathrm{CI}$ $3.74-12.45 ; p=0.042 ; \Delta$ NIHSS $\geq 3$ : OR $5.76 ; 95 \%$ CI 3.98-8.27; $p=0.013$ ) were observed, whereas no associations were found for SC-treated patients (all with $p>0.05$ ). The results of the logistic regression analysis are presented in table 3. Furthermore, an $\mathrm{mRS}$ reduction shift analysis yielded comparable results (table e-2, links.lww.com/WNL/A440). A visual representation of the clinical course of the NIHSS score between admission and 24 hours thereafter is provided in figure e-5 (links.lww.com/WNL/A439).

\section{Discussion}

This study investigated the predictive value of waveletCTAbased detection of distal vessel occlusions that are not evident on CTA in patients with acute ischemic stroke. Patients with such occlusions who received IVT, in contrast to patients who received only SC, had more favorable morphologic outcomes independent of established predictors such as admission NIHSS score, early ischemic changes, or CBF-CBV mismatch. Regarding functional outcome, IVTtreated patients with waveletCTA-detected occlusions, as opposed to patients treated with SC, had more favorable primary and secondary functional outcomes, particularly a better neurologic improvement measured by the difference in the NIHSS scores between admission and 24 hours thereafter. 
Table 1 Patient characteristics stratified by IV thrombolysis treatment and occlusion detection

\begin{tabular}{|c|c|c|c|c|c|}
\hline & \multicolumn{2}{|c|}{ IV thrombolysis ( $n=57$ ) } & \multicolumn{2}{|c|}{ Supportive care $(n=50)$} & \multirow[b]{2}{*}{$p$ Value } \\
\hline & Occlusion $(n=35)$ & No occlusion $(n=22)$ & Occlusion $(n=23)$ & No occlusion $(n=27)$ & \\
\hline \multicolumn{6}{|l|}{ Patient data } \\
\hline Age, y & $75(67-83)$ & $71(67-79)$ & $73(64-79)$ & $76(70-80)$ & 0.820 \\
\hline Sex, male, n (\%) & $24(68.6)$ & $11(50.0)$ & $16(69.6)$ & $15(55.5)$ & 0.391 \\
\hline Time from symptom onset ${ }^{a}$ & $105(90-150)$ & $153(105-210)$ & $105(60-210)$ & $135(113-233)$ & 0.156 \\
\hline MCA stroke, n (\%) & $29(82.9)$ & $17(77.3)$ & $18(78.3)$ & $20(74.1)$ & 0.457 \\
\hline \multicolumn{6}{|l|}{ Imaging data } \\
\hline Early ischemic changes, n (\%) & $10(28.6)$ & $6(27.3)$ & $8(34.8)$ & $9(33.3)$ & 0.393 \\
\hline ASPECTS & $10(9-10)$ & $10(9-10)$ & $9(9-10)$ & $10(9-10)$ & 0.431 \\
\hline Extracranial $\geq 70 \%$ stenosis, $n(\%)$ & $2(5.7)$ & $1(4.5)$ & $1(4.3)$ & $1(3.7)$ & 0.884 \\
\hline CBF deficit volume & $24(13-39)$ & $8(2-18)$ & $18(13-30)$ & $6(3-13)$ & $<0.001^{\mathrm{b}}$ \\
\hline CBV deficit volume & $5(2-11)$ & $3(2-6)$ & $6(2-16)$ & $2(2-5)$ & 0.122 \\
\hline CBF-CBV mismatch, \% & $77(66-87)$ & $68(48-81)$ & $66(50-78)$ & $63(31-80)$ & 0.124 \\
\hline Final infarction volume & $3(2-15)$ & $7(2-22)$ & $14(6-38)$ & $3(2-8)$ & $0.011^{b}$ \\
\hline \multicolumn{6}{|l|}{ Functional data } \\
\hline Admission NIHSS & $6(5-9)$ & $9(4-14)$ & $6(3-9)$ & $4(2-7)$ & 0.436 \\
\hline 24-h NIHSS & $2(1-3)$ & $7(3-13)$ & $7(5-9)$ & $3(1-5)$ & $0.001^{b}$ \\
\hline$\Delta$ NIHSS (admission to $24 \mathrm{~h}$ ) & $4(3-6)$ & $0(0-2)$ & $-1(-2-0)$ & $0(0-1)$ & $<0.001^{\mathrm{b}}$ \\
\hline Premorbid mRS & $0(0-1)$ & $0(0-1)$ & $1(0-3)$ & $0(0-0)$ & 0.079 \\
\hline Discharge $\mathrm{mRS}^{\mathrm{a}}$ & $1(1-2)$ & $3(2-4)$ & $3(2-4)$ & $2(1-4)$ & $0.015^{b}$ \\
\hline 90-d mRs & $1(0-1)$ & $1(1-3)$ & $3(2-6)$ & $1(0-2)$ & $0.031^{b}$ \\
\hline \multicolumn{6}{|l|}{ Cardiovascular risk factors, n (\%) } \\
\hline Arterial hypertension & $25(71.4)$ & $11(50.0)$ & $14(63.6)$ & $19(76.0)$ & 0.245 \\
\hline Atrial fibrillation & $10(28.6)$ & $6(27.3)$ & $10(45.5)$ & $6(24.0)$ & 0.396 \\
\hline Smoking & $13(37.1)$ & $3(13.6)$ & $8(36.4)$ & $9(36.0)$ & 0.238 \\
\hline Hypercholesterolemia & $9(25.7)$ & $7(31.8)$ & $5(22.7)$ & $6(24.0)$ & 0.905 \\
\hline Diabetes mellitus & $7(20.0)$ & $4(18.2)$ & $6(27.3)$ & $7(28.0)$ & 0.791 \\
\hline \multicolumn{6}{|l|}{ Etiology of stroke, n (\%) } \\
\hline Cardioembolic & $11(31.4)$ & $10(45.5)$ & $10(43.5)$ & $9(33.3)$ & 0.638 \\
\hline Arterioarterial & $13(37.1)$ & $9(40.9)$ & $6(26.1)$ & $9(33.3)$ & 0.743 \\
\hline Other determined & $1(2.9)$ & $0(0.0)$ & $3(13.0)$ & $2(7.4)$ & NA \\
\hline Undetermined & $10(28.6)$ & $3(13.6)$ & $4(17.4)$ & $7(25.9)$ & 0.520 \\
\hline \multicolumn{6}{|l|}{ Complications, n (\%) } \\
\hline Hemorrhagic infarction & & & & & NA \\
\hline Type 1 & $2(5.7)$ & $1(4.5)$ & $0(0.0)$ & $3(11.1)$ & \\
\hline Type 2 & $2(5.7)$ & $0(0.0)$ & $3(13.0)$ & $1(3.7)$ & \\
\hline Parenchymal hematoma & & & & & NA \\
\hline Type 1 & $0(0.0)$ & $1(4.5)$ & $1(4.3)$ & $1(3.7)$ & \\
\hline
\end{tabular}


Table 1 Patient characteristics stratified by IV thrombolysis treatment and occlusion detection (continued)

\begin{tabular}{|c|c|c|c|c|c|}
\hline & \multicolumn{2}{|c|}{ IV thrombolysis $(n=57)$} & \multicolumn{2}{|c|}{ Supportive care $(n=50)$} & \multirow[b]{2}{*}{$p$ Value } \\
\hline & Occlusion $(n=35)$ & No occlusion $(n=22)$ & Occlusion $(n=23)$ & No occlusion $(n=27)$ & \\
\hline Type 2 & $0(0.0)$ & $0(0.0)$ & $0(0.0)$ & $0(0.0)$ & \\
\hline Extraischemic ICH & $1(2.9)$ & $0(0.0)$ & $1(4.3)$ & $2(7.4)$ & NA \\
\hline Space-occupying edema & $0(0.0)$ & $0(0.0)$ & $1(4.3)$ & $1(3.7)$ & NA \\
\hline
\end{tabular}

Abbreviations: ASPECTS = Alberta Stroke Program Early CT Score; CBF = cerebral blood flow; CBV = cerebral blood volume; ICH = intracranial hemorrhage; MCA = middle cerebral artery; $m R S=$ modified Rankin Scale; NA = not applicable; NIHSS = NIH Stroke Scale.

Values presented are $\mathrm{n}(\%)$ for categorical variables and median (interquartile range) for ordinal and continuous variables. Proportion analysis tests for categorical variables were performed using the $\chi^{2}$ test. Nonparametric tests for continuous variables were performed using analysis of variance and for ordinal variables using the Kruskal-Wallis test.

a Missing values: time from symptom onset 31/107, discharge mRS 4/107, 24-hour NIHSS 4/107, 90-day mRS 17/107.

${ }^{b}$ Statistically significant.

Regardless of the recent success of endovascular thrombectomy in patients with large-vessel occlusions, IVT is still the mainstay of ischemic stroke therapy in the majority of patients. ${ }^{1,17}$ Stroke patients without evidence of a vessel occlusion are known to have better functional outcomes. ${ }^{36,37}$ However, there is no consensus on the differential treatment effect of IVT in patients with compared to patients without vessel occlusions. While earlier studies suggest that patients without evidence of vessel occlusions benefit from IVT, ${ }^{2,3} 2$ recent, large studies were not able to find a significant benefit of IVT for this patient subgroup. ${ }^{4,5}$ Another recent study on patients with small perfusion deficits of $<15 \mathrm{~mL}$, of which only
$15 \%$ showed an occlusion, also showed no benefit of IVT regarding functional outcome. ${ }^{38}$

The evaluation of patients with waveletCTA for the presence or absence of distal vessel occlusions, however, allows further classification of patients with negative CTA. The differential treatment effect of IVT in patients with compared to patients without waveletCTA-detected occlusions in our study could represent a possible explanation for the differences that were observed in the above-mentioned studies. From a pathophysiologic point of view, the vessel-occluding clot as the primary target of a thrombolytic therapy

Table 2 Distal vessel occlusions and morphologic outcome in treatment subgroups

\begin{tabular}{|c|c|c|c|c|}
\hline \multirow[b]{3}{*}{ Independent variable } & \multicolumn{4}{|c|}{ Dependent morphologic outcome variables } \\
\hline & \multicolumn{2}{|l|}{ IV thrombolysis } & \multicolumn{2}{|l|}{ Supportive care } \\
\hline & $\beta(95 \% \mathrm{Cl})$ & $p$ Value & $\beta(95 \% \mathrm{Cl})$ & $p$ Value \\
\hline \multirow[t]{2}{*}{ Distal vessel occlusion ${ }^{a}$} & FIV & & & \\
\hline & $-7.43(-14.86,1.82)$ & 0.107 & $8.76(-11.51,29.02)$ & 0.386 \\
\hline \multirow[t]{2}{*}{ Distal vessel occlusion ${ }^{a}$} & $\begin{array}{l}\text { Ratio of FIV to admission total } \\
\text { ischemic volume (FIV/CBF) }\end{array}$ & & & \\
\hline & $-1.43(-1.96,-0.83)$ & $0.001^{c}$ & $0.47(-0.06,1.01)$ & 0.081 \\
\hline \multirow[t]{2}{*}{ Distal vessel occlusion ${ }^{a}$} & $\begin{array}{l}\text { Ratio of FIV to admission core } \\
\text { ischemic volume (FIV/CBV) }\end{array}$ & & & \\
\hline & $-1.92(-4.76,0.92)$ & 0.080 & $1.01(0.80,2.81)$ & 0.264 \\
\hline \multirow[t]{2}{*}{ Distal vessel occlusion ${ }^{a}$} & $\begin{array}{l}\text { Ratio of final penumbral infarction } \\
\text { volume to admission } \\
\text { penumbral ischemic volume } \\
([\mathrm{FIV}-\mathrm{CBV}] /[\mathrm{CBF}-\mathrm{CBV}])^{\mathrm{e}}\end{array}$ & & & \\
\hline & $-4.54(-7.02,-2.07)$ & $0.001^{c}$ & $1.86(-1.63,5.35)$ & 0.285 \\
\hline \multicolumn{5}{|c|}{$\begin{array}{l}\text { Abbreviations: } \mathrm{CBF}=\text { cerebral blood flow; } \mathrm{CBV}=\text { cerebral blood volume; } \mathrm{Cl}=\text { confidence interval; FIV = final infarction volume. } \\
\text { A multivariate linear regression analysis was performed for the indicated morphologic outcome parameters for the patients receiving IV thrombolysis or } \\
\text { supportive care. } \\
\text { a Adjusted for age, sex, admission NIH Stroke Scale, early ischemic changes, size of the perfusion deficit on CBF and CBV, as well as the percentage of CBF-CBV } \\
\text { mismatch. } \\
\text { b Total ischemic volume is based on the size of the CBF perfusion deficit. } \\
\text { 'Statistically significant. } \\
\text { d Core ischemic volume is based on the size of the CBV perfusion deficit. } \\
\text { e Penumbral ischemic volume is calculated as the size of the CBF minus the CBV perfusion deficit. }\end{array}$} \\
\hline
\end{tabular}




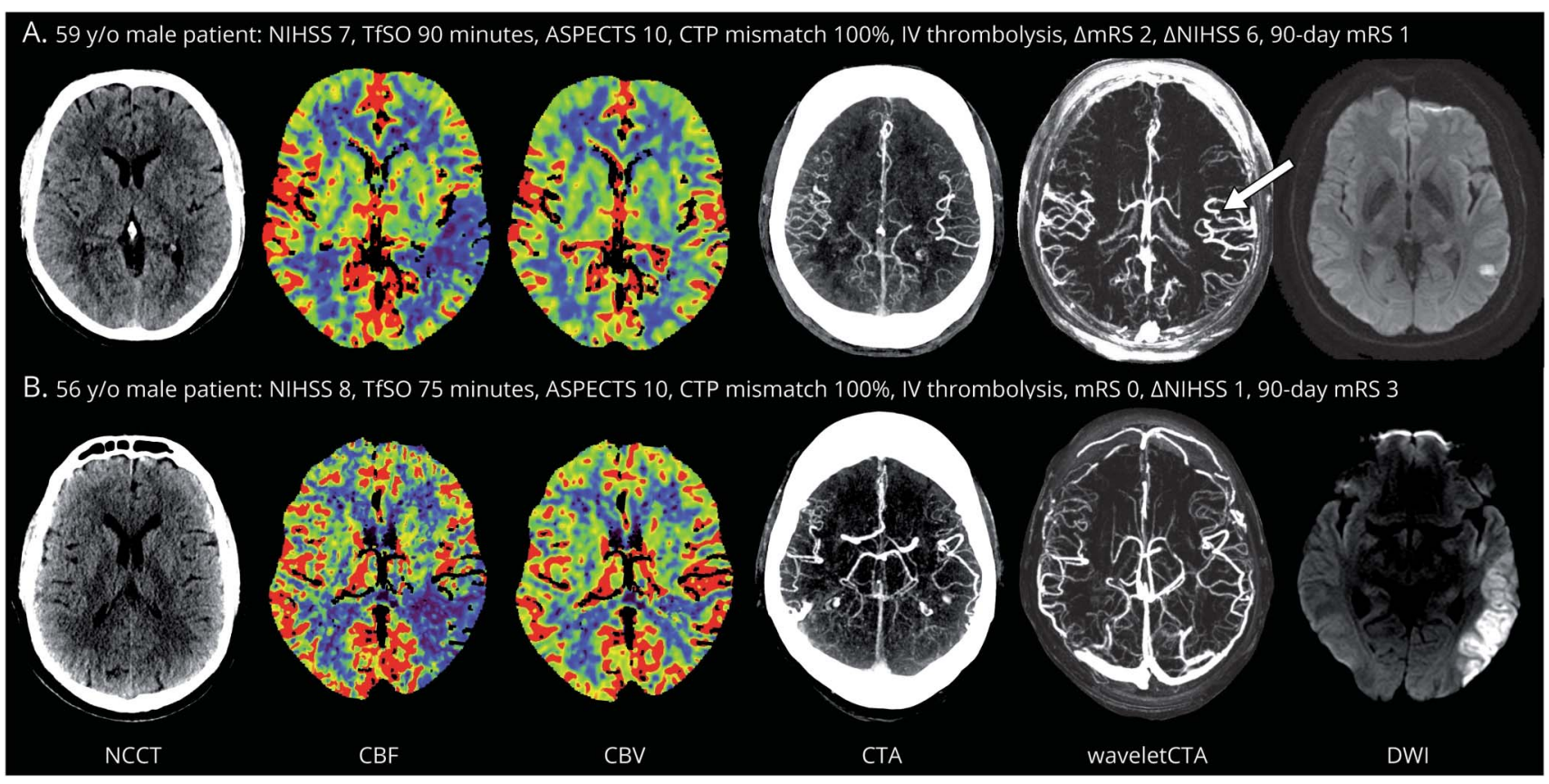

(A) A 59-year-old man and (B) a 56-year-old man presented with a right-sided hemiparesis and similar NIHSS scores on admission, TfSO, ASPECTS, and CTP mismatch. Both patients received immediate IV thrombolysis treatment. In patient A, an M3 segment vessel occlusion not evident on CTA was identified using waveletCTA (white arrow). In contrast, no occlusions were evident in patient B, neither on CTA nor on waveletCTA. Patient A showed a favorable morphologic and clinical outcome (final infarction volume: $1.2 \mathrm{~mL}$; CBF deficit volume: $16.5 \mathrm{~mL} ; 90$-day mRS score: 1). Patient B demonstrated an unfavorable morphologic as well as clinical outcome (final infarction volume: $25.4 \mathrm{~mL}$; CBF deficit volume: $28.2 \mathrm{~mL}$; 90 -day mRS score: 3 ). CTA and waveletCTA are presented as maximum intensity projections. ASPECTS = Alberta Stroke Program Early CT Score; $C B F=$ cerebral blood flow; $C B V=$ cerebral blood volume; $C T A=C T$ angiography; $\mathrm{CTP}=\mathrm{CT}$ perfusion; $\mathrm{DWI}$ = diffusion-weighted imaging; $\mathrm{mRS}$ = modified Rankin Scale; $\mathrm{NCCT}$ = noncontrast CT; NIHSS = NIH Stroke Scale; TfSO = time from symptom onset.

represents a possible reason for the larger IVT treatment effect observed in our study.

Of note, waveletCTA-detected occlusions in IVT-treated patients were associated with favorable morphologic outcome parameters FIV/CBF and (FIV - CBV)/(CBF - CBV), which both consider the ischemic penumbra, but not FIV/ $\mathrm{CBV}$, which only accounts for the progression of the ischemic core to the final infarction. This could be explained by the pathophysiologic concept that distinguishes irreversibly

Table 3 Distal vessel occlusions and functional outcome in treatment subgroups

\begin{tabular}{|c|c|c|c|c|}
\hline \multirow[b]{3}{*}{ Independent variable } & \multicolumn{4}{|c|}{ Dependent functional outcome variables } \\
\hline & \multicolumn{2}{|l|}{ IV thrombolysis } & \multicolumn{2}{|l|}{ Supportive care } \\
\hline & Adjusted OR $(95 \% \mathrm{Cl})$ & $p$ Value & Adjusted OR (95\% Cl) & $p$ Value \\
\hline \multirow[t]{2}{*}{ Distal vessel occlusion ${ }^{a}$} & $90-\mathrm{d} m R S \leq 2$ & & & \\
\hline & $7.68(4.33,11.51)$ & $0.039^{b}$ & $1.71(0.46,6.37)$ & 0.362 \\
\hline \multirow[t]{2}{*}{ Distal vessel occlusion ${ }^{a}$} & Discharge $\mathrm{mRS} \leq 2$ & & & \\
\hline & $8.33(3.74,12.45)$ & $0.042^{\mathrm{b}}$ & $0.64(0.02,17.62)$ & 0.811 \\
\hline \multirow[t]{2}{*}{ Distal vessel occlusion ${ }^{a}$} & $\begin{array}{l}\text { NIHSS improvement from } \\
\text { admission to } 24 \mathrm{~h}(\Delta \text { NIHSS } \geq 3)\end{array}$ & & & \\
\hline & $5.76(3.98,8.27)$ & $0.013^{b}$ & $0.25(0.01,18.77)$ & 0.258 \\
\hline
\end{tabular}

Abbreviations: $\mathrm{Cl}=$ confidence interval; $\mathrm{mRS}=$ modified Rankin Scale; NIHSS = NIH Stroke Scale; OR = odds ratio.

A binary logistic regression analysis was performed for the indicated functional outcome parameters for the patients receiving IV thrombolysis or supportive care.

${ }^{a}$ Adjusted for age, sex, admission NIHSS, early ischemic changes, size of the perfusion deficit on cerebral blood flow (CBF) and cerebral blood volume (CBV), as well as the percentage of CBF-CBV mismatch.

b Statistically significant. 
damaged brain tissue (ischemic core) and potentially salvageable tissue at risk (ischemic penumbra). Integrating our observations in this concept, IVT might therefore be unable to influence the progression of the ischemic core to the final infarction irrespective of available therapeutic targets in the form of vessel occlusions.

Despite proven ischemic lesions on CTP imaging, we only observed underlying vessel occlusions in about half of the patients. Possible explanations are that (1) spontaneous recanalization has already occurred by the time of $\operatorname{admission}^{14,15}$ or that (2) waveletCTA misses smaller occlusions because of the limited spatial resolution of contemporary CT imaging. The presence of a high-grade extracranial stenosis was very low in the study population and evenly distributed across the subgroups. We therefore consider it unlikely that the ischemic lesions could have resulted from an extracranial stenosis.

The persistence of perfusion lesions despite macrovascular recanalization, also termed the no-reflow phenomenon, has been observed by several basic science studies on ischemic stroke $^{7,11,12}$ as well as in human clinical ischemic stroke trials. $^{8-10}$ It is known that oxidative stress signals resulting from hypoperfusion lead to microvascular obstruction mediated by capillary pericytes. ${ }^{11,12}$ Targeting this mechanism conferred neuroprotection and improved outcomes in animal models of ischemic stroke, ${ }^{13}$ but the relevance in humans remains unclear. In this pathophysiologic context, the added value of waveletCTA may be to triage patients with acute ischemic perfusion lesions based on the presence of a macrovascular occlusion, which represents the main target of IVT.

Despite the well-proven benefits of IVT, undertreatment of patients with stroke remains an issue. ${ }^{39}$ A recent survey demonstrated that CTA findings are important influencing factors for $41 \%$ of North American neurologists in their decision of IVT administration. ${ }^{40}$ Thus, the identification of additional occlusions using angiographic CTP postprocessing techniques may support the decision-making process to increase diagnostic confidence and the use of IVT. However, in light of equivocal data on the benefit of IVT in patients without evidence of occlusions on CTA, this aspect is not considered in the current guidelines on stroke management. ${ }^{16,17}$

There are limitations to this study that need to be considered when interpreting the results. First, this is a single-center study with a limited number of patients. However, because our results were consistent among different morphologic and functional parameters, we assume that larger studies will come to similar conclusions. Second, the retrospective design of our study makes it potentially prone to selection bias. Nonetheless, all enrolled patients were recruited from a prospectively collected stroke registry, and standardized stroke protocols were applied to minimize this potential bias. Third, based on the study design, no diagnostic standard of reference for
waveletCTA-detected occlusions is available. However, in a previous diagnostic accuracy study using stroke patients with CTA-proven occlusions and control patients without follow-up infarction, waveletCTA demonstrated high specificity for occlusion detection. ${ }^{24}$ On the other hand, it should be acknowledged that the sensitivity of waveletCTA may be limited and that other reconstruction techniques may yield similar results. Fourth, expert visual delineation of CTP lesions was applied for volumetric assessment as the manufacturer's automated analysis was not applicable to all vascular territories, and misclassification of medium-sized lesions was observed. Because no threshold could be applied in this approach, a differentiation from benign oligemic changes is potentially limited. Yet, all patients were included on the basis of a follow-up-confirmed infarction. Moreover, reliability and validity of this approach has been shown by several studies. ${ }^{29-31}$ Nevertheless, further studies with larger patient cohorts will need to be conducted to validate the prognostic relevance of distal vessel occlusions with respect to efficacy and safety of IVT.

In our study on patients with acute ischemic stroke, distal vessel occlusions as detected using wavelet-based CTP postprocessing predicted a favorable response to IVT and may have potential implications in clinical decisionmaking.

\section{Author contributions}

W.G.K.: study design, data analysis and interpretation, draft and revision of manuscript. M.P.F.: study design, data analysis and interpretation. W.H.S.: study design, data analysis and interpretation, revision of manuscript. C.H.: data analysis and interpretation, revision of manuscript. P.S.: data analysis and interpretation, revision of manuscript. L.T.R.: data analysis and interpretation. W.P.F.: data analysis and interpretation, revision of manuscript. B.O.S.: data analysis and interpretation, revision of manuscript. F.G.M.: data analysis and interpretation, revision of manuscript. F.D.: revision of manuscript. B.E.-W.: revision of manuscript. M.F.R.: revision of manuscript. K.M.T.: study design, data analysis and interpretation, draft and revision of manuscript.

\section{Study funding}

No targeted funding reported.

\section{Disclosure}

The authors report no disclosures relevant to the manuscript. Go to Neurology.org/N for full disclosures.

Received August 23, 2017. Accepted in final form February 26, 2018.

\section{References}

1. The National Institute of Neurological Disorders and Stroke rt-PA Stroke Study Group. Tissue plasminogen activator for acute ischemic stroke. N Engl J Med 1995; 333:1581-1587.

2. Mikulik R, Goldemund D, Reif M, Aulicky P, Krupa P. Outcome of patients with negative $\mathrm{CT}$ angiography results for arterial occlusion treated with intravenous thrombolysis. Stroke 2009;40:868-872.

3. Lahoti S, Gokhale S, Caplan L, et al. Thrombolysis in ischemic stroke without arterial occlusion at presentation. Stroke 2014;45:2722-2727. 
4. Mair G, von Kummer R, Adami A, et al. Arterial obstruction on computed tomographic or magnetic resonance angiography and response to intravenous thrombolytics in ischemic stroke. Stroke 2016;48:353-360.

5. Medlin F, Amiguet M, Vanacker P, Michel P. Influence of arterial occlusion on outcome after intravenous thrombolysis for acute ischemic stroke. Stroke 2015;46: 126-131.

6. De Silva DA, Churilov L, Olivot JM, et al. Greater effect of stroke thrombolysis in the presence of arterial obstruction. Ann Neurol 2011;70:601-605.

7. Ames A III, Wright RL, Kowada M, Thurston JM, Majno G. Cerebral ischemia. II. The no-reflow phenomenon. Am J Pathol 1968;52:437-453.

8. Soares BP, Chien JD, Wintermark M. MR and CT monitoring of recanalization, reperfusion, and penumbra salvage: everything that recanalizes does not necessarily reperfuse! Stroke 2009;40:S24-S27.

9. De Silva DA, Fink JN, Christensen S, et al. Assessing reperfusion and recanalization as markers of clinical outcomes after intravenous thrombolysis in the Echoplanar Imaging Thrombolytic Evaluation Trial (EPITHET). Stroke 2009;40:2872-2874.

10. Horsch AD, Dankbaar JW, Niesten JM, et al. Predictors of reperfusion in patients with acute ischemic stroke. AJNR Am J Neuroradiol 2015;36:1056-1062.

11. Yemisci M, Gursoy-Ozdemir Y, Vural A, Can A, Topalkara K, Dalkara T. Pericyte contraction induced by oxidative-nitrative stress impairs capillary reflow despite successful opening of an occluded cerebral artery. Nat Med 2009;15:1031-1037.

12. Hall CN, Reynell C, Gesslein B, et al. Capillary pericytes regulate cerebral blood flow in health and disease. Nature 2014;508:55-60.

13. Dalkara T, Arsava EM. Can restoring incomplete microcirculatory reperfusion improve stroke outcome after thrombolysis? J Cereb Blood Flow Metab 2012;32: 2091-2099.

14. Dalal PM, Shah PM, Sheth SC, Deshpande CK. Cerebral embolism: angiographic observations on spontaneous clot lysis. Lancet 1965;1:61-64.

15. Babikian VL, Caplan LR. Brain embolism is a dynamic process with variable characteristics. Neurology 2000;54:797-801.

16. Jauch EC, Saver JL, Adams HP Jr, et al. Guidelines for the early management of patients with acute ischemic stroke: a guideline for healthcare professionals from the American Heart Association/American Stroke Association. Stroke 2013;44: 870-947.

17. Powers WJ, Derdeyn CP, Biller J, et al. 2015 American Heart Association/American Stroke Association focused update of the 2013 guidelines for the early management of patients with acute ischemic stroke regarding endovascular treatment: a guideline for healthcare professionals from the American Heart Association/American Stroke Association. Stroke 2015;46:3020-3035.

18. Wildermuth S, Knauth M, Brandt T, Winter R, Sartor K, Hacke W. Role of CT angiography in patient selection for thrombolytic therapy in acute hemispheric stroke. Stroke 1998;29:935-938.

19. Smit EJ, Vonken EJ, van der Schaaf IC, et al. Timing-invariant reconstruction for deriving high-quality CT angiographic data from cerebral CT perfusion data. Radiology 2012;263:216-225.

20. Havla L, Schneider M, Thierfelder KM, et al. Validation of a method to differentiate arterial and venous vessels in CT perfusion data using linear combinations of quantitative time-density curve characteristics. Eur Radiol 2015;25:2937-2944.

21. Smit EJ, Vonken EJ, van Seeters T, et al. Timing-invariant imaging of collateral vessels in acute ischemic stroke. Stroke 2013;44:2194-2199.
22. Wintermark M, Luby M, Bornstein NM, et al. International survey of acute stroke imaging used to make revascularization treatment decisions. Int J Stroke 2015;10: 759-762.

23. Frolich AM, Psychogios MN, Klotz E, Schramm R, Knauth M, Schramm P. Angiographic reconstructions from whole-brain perfusion CT for the detection of large vessel occlusion in acute stroke. Stroke 2012;43:97-102.

24. Kunz WG, Sommer WH, Havla L, et al. Detection of single-phase CTA occult vessel occlusions in acute ischemic stroke using CT perfusion-based wavelet-transformed angiography. Eur Radiol 2017;27:2657-2664.

25. Havla L, Thierfelder KM, Beyer SE, Sommer WH, Dietrich O. Wavelet-based calculation of cerebral angiographic data from time-resolved $\mathrm{CT}$ perfusion acquisitions. Eur Radiol 2015;25:2354-2361.

26. Kunz WG, Schuler F, Sommer WH, et al. Wavelet-based angiographic reconstruction of computed tomography perfusion data: diagnostic value in cerebral venous sinus thrombosis. Invest Radiol 2017;52:302-309.

27. Pexman JH, Barber PA, Hill MD, et al. Use of the Alberta Stroke Program early CT Score (ASPECTS) for assessing CT scans in patients with acute stroke. AJNR Am J Neuroradiol 2001;22:1534-1542.

28. Wardlaw JM, Mielke O. Early signs of brain infarction at CT: observer reliability and outcome after thrombolytic treatment—systematic review. Radiology 2005;235:444-453.

29. Thierfelder KM, Sommer WH, Baumann AB, et al. Whole-brain CT perfusion: reliability and reproducibility of volumetric perfusion deficit assessment in patients with acute ischemic stroke. Neuroradiology 2013;55:827-835.

30. Muir KW, Halbert HM, Baird TA, McCormick M, Teasdale E. Visual evaluation of perfusion computed tomography in acute stroke accurately estimates infarct volume and tissue viability. J Neurol Neurosurg Psychiatry 2006;77:334-339.

31. Mouridsen K, Nagenthiraja K, Jonsdottir KY, et al. Acute stroke: automatic perfusion lesion outlining using level sets. Radiology 2013;269:404-412.

32. Campbell BC, Christensen S, Tress BM, et al. Failure of collateral blood flow is associated with infarct growth in ischemic stroke. J Cereb Blood Flow Metab 2013;33: $1168-1172$

33. Kamalian S, Kemmling A, Borgie RC, et al. Admission insular infarction $>25 \%$ is the strongest predictor of large mismatch loss in proximal middle cerebral artery stroke. Stroke 2013;44:3084-3089.

34. Campbell BC, Mitchell PJ, Kleinig TJ, et al. Endovascular therapy for ischemic stroke with perfusion-imaging selection. N Engl J Med 2015;372:1009-1018.

35. Lin L, Cheng X, Bivard A, Levi CR, Dong Q, Parsons MW. Quantifying reperfusion of the ischemic region on whole-brain computed tomography perfusion. J Cereb Blood Flow Metab 2017;37:2125-2136.

36. Arnold M, Nedeltchev K, Brekenfeld C, et al. Outcome of acute stroke patients without visible occlusion on early arteriography. Stroke 2004;35:1135-1138.

37. Sims JR, Rordorf G, Smith EE, et al. Arterial occlusion revealed by CT angiography predicts NIH stroke score and acute outcomes after IV tPA treatment. AJNR Am J Neuroradiol 2005;26:246-251.

38. Bivard A, Lou M, Levi CR, et al. Too good to treat? Ischemic stroke patients with small CT perfusion lesions may not benefit from thrombolysis. Ann Neurol 2016;80: 286-293.

39. Messe SR, Khatri P, Reeves MJ, et al. Why are acute ischemic stroke patients not receiving IV tPA? Results from a national registry. Neurology 2016;87:1565-1574.

40. Shamy MC, Pugliese M, Meisel K, et al. How patient demographics, imaging, and beliefs influence tissue-type plasminogen activator use: a survey of North American neurologists. Stroke 2016;47:2051-2057. 


\title{
Effect of stroke thrombolysis predicted by distal vessel occlusion detection
}

\author{
Wolfgang G. Kunz, MD, Matthias P. Fabritius, MD, Wieland H. Sommer, MD, MPH, Christopher Höhne, MD, \\ Pierre Scheffler, MD, Lukas T. Rotkopf, Wolfgang P. Fendler, MD, Bastian O. Sabel, MD, Felix G. Meinel, MD, \\ Franziska Dorn, MD, Birgit Ertl-Wagner, MD, MHBA, Maximilian F. Reiser, MD, \\ and Kolja M. Thierfelder, MD, MSc
}

Cite as: Neurology ${ }^{\circledR}$ 2018;90:e1742-e1750. doi:10.1212/WNL.0000000000005519

\author{
Correspondence \\ Dr. Kunz \\ wolfgang.kunz@med.Imu.de
}

\section{Study question}

Can enhanced distal vessel occlusion detection with CT perfusion (CTP)-based wavelet-transformed angiography (waveletCTA) predict responses to intravenous thrombolysis (IVT) in ischemic stroke patients who test negative for vessel occlusions in CT angiography (CTA)?

\section{Summary answer}

Distal vessel occlusions detected with waveletCTA independently predict favorable responses to IVT in ischemic stroke patients with negative CTA results.

\section{What is known and what this paper adds}

IVT provides reperfusion of ischemic tissue by targeting occluded vessels. Prior studies suggested larger treatment benefits for patients with evidence of occlusion. This study shows that by providing enhanced detection of vessel occlusions, waveletCTA can predict the response to IVT in ischemic stroke patients with negative CTA results.

\section{Participants and setting}

This study analyzed 107 patients with acute ischemic stroke who underwent CT imaging at Munich's Ludwig-MaximiliansUniversity Hospital between December 2012 and December 2016. This group included 57 patients treated with IVT (61\% male) and 50 patients treated with supportive care (SC; $62 \%$ male). All participants exhibited cerebral blood flow (CBF) deficits on CTP scans and had negative CTA results.

\section{Design, size, and duration}

The waveletCTA images obtained by processing CTP scans were reviewed by 2 experienced radiologists to identify vessel occlusions. The radiologists were blinded to clinical information.

\section{Primary outcomes}

The primary morphologic outcome was the final infarction volume divided by the initial CBF deficit volume, with smaller ratios being considered more favorable. The primary functional outcome was the modified Rankin Scale (mRS) score after 90 days, with scores $\leq 2$ being defined as favorable.
Table Predictive power of waveletCTA-detected distal vessel occlusions for favorable morphologic and functional outcomes in the context of IVT treatment

\begin{tabular}{lll}
\hline $\begin{array}{l}\text { Treatment } \\
\text { group }\end{array}$ & $\begin{array}{l}\boldsymbol{\beta}(95 \% \mathrm{CI}) \\
\text { for FIV/CBF }\end{array}$ & $\begin{array}{l}\text { Adjusted OR }(95 \% \mathrm{CI}) \\
\text { for } 90-\mathrm{d} \text { mRS score } \leq 2\end{array}$ \\
\hline IVT group & $-1.43(-1.96$ to -0.83$)$ & $7.68(4.33-11.51)$ \\
\hline SC group & $0.47(-0.06$ to 1.01$)$ & $1.71(0.46-6.37)$ \\
\hline
\end{tabular}

Abbreviations: $\mathrm{CBF}=$ cerebral blood flow; $\mathrm{Cl}=$ confidence interval; FIV = final infarction volume; IVT = intravenous thrombolysis; $\mathrm{mRS}=$ modified Rankin Scale; OR = odds ratio; $\mathrm{SC}$ = supportive care; waveletCTA = CT perfusionbased wavelet-transformed angiography.

\section{Main results and the role of chance}

The waveletCTA images revealed distal occlusions in 58 (54\%) participants. In the IVT-treated patients, waveletCTAdetected distal vessel occlusions predicted favorable morphologic outcomes $(p=0.001)$ and favorable functional outcomes $(p=0.039)$. In the SC-treated patients, waveletCTA-detected distal vessel occlusions did not predict favorable morphologic outcomes $(p=0.081)$ or favorable functional outcomes $(p=0.362)$.

\section{Bias, confounding, and other reasons for caution}

This study's retrospective nature made it prone to selection bias. As invasive angiography is not clinically indicated in this population, no diagnostic standard of reference for wavelet CTA-detected occlusions was available.

\section{Generalizability to other populations}

This was a single-center study with a small group of patients, so the generalizability of the results may be limited.

\section{Study funding/potential competing interests}

This study received no funding, and the authors report no competing interests. Go to Neurology.org/ $\mathrm{N}$ for full disclosures. 